\title{
A secure and privacy preserving iris biometric authentication scheme with matrix transformation
}

\begin{abstract}
Biometric authentication is the use of unique human features to provide secure, reliable, friendly and convenient access to an environment or a computer installation. However, the use of biometrics as a means of authentication exposes legitimate users to security threats, privacy attacks and loss of identity. This paper proposes and implements a novel noninvertible transformation technique known as matrix transformation. Matrix transformation is a simple but powerful and effective method to achieve template revocability and prevent the recovery of original biometric data from secured templates. The approach provides a high level template security and user privacy. It is also robust against replay attack, cross matching and loss of identity.
\end{abstract}

Keyword: Authentication; Biometric; Non-invertible; Transformation; Security; Privacy 